\section{Individual differences and the use of nonspecifying variables in learning to perceive distance and size: Comments on McConnell, Muchisky, and Bingham (1998)}

\author{
DAVID M. JACOBS and CLAIRE F. MICHAELS \\ Vrije Universiteit, Amsterdam, The Netherlands
}

McConnell, Muchisky, and Bingham (1998) showed that observers are able to judge the distance and size of falling, rolling, and swinging balls and that performance improves after practice with feedback. They concluded that observers use information that specifies the spatial scales of the different event types-namely, event duration in combination with event-specific constants. The improvement was interpreted as the calibration of the event-specific constants. We argue that their analyses should have considered the use of optical variables that do not specify the to-be-perceived metrics and individual differences in variable use. Furthermore, we propose convergence on the more useful variables as an alternative explanation for the observed improvement. The viability of these arguments is demonstrated with an experiment in which participants are trained with feedback to judge the distance and size offreely falling balls.

How are observers able to perceive distance and size while definite spatial metrics are lost in the mapping from objects and events to optical patterns? McConnell, Muchisky, and Bingham (1998) showed that the spatial scales of several event types are related lawfully to the event durations, but they also showed that the particular relation between temporal information and spatial scale is different for different event types. This led them to suggest that, in order to perceive spatial metrics, observers might recognize events and then use the appropriate relation between temporal information and spatial scale.

McConnell et al. (1998) considered perception of the distance and size of balls that were freely falling, rolling down inclines, or swinging on the end of pendulums. Under the boundary condition of negligible drag, the distance $(d)$ covered by a falling ball is $d=(\mathrm{g} / 2) t^{2}$, in which $g$ is the gravitational constant $\left(9.8 \mathrm{~m} / \mathrm{sec}^{2}\right)$ and $t$ the duration of the fall. For a (solid, homogenous) ball rolling down an incline, the distance $(d)$ along the incline is $d=$

The Netherlands Organization for Scientific Research (NWO) is gratefully acknowledged for funding this project. This research was conducted while D.M.J. was supported by Grant 575-12-070 from this organization's Foundation for Behavioural and Educational Sciences, awarded to C.F.M. We also thank Daniel McConnell and Frank Zaal for comments on earlier versions of this article, Martijn Heijmans, Mariëtte Jansen, Remco Kortenoeven, and Diane Breedijk for running the experiment, and Harold Sedgwick, Geoffrey Bingham, and an anonymous reviewer for helpful reviews. Correspondence concerning this article should be addressed to D. M. Jacobs, Faculty of Human Movement Science, Vrije Universiteit, van der Boechorststraat 9, 1081 BT Amsterdam, The Netherlands (e-mail: d_jacobs@fbw.vu.nl).
$(5 \mathrm{~g} / 14) \sin (\theta) t^{2}$, where $\theta$ is the angle of the slope from the horizontal.1,2 Finally, the length $(l)$ of a pendulum that swings with a small amplitude is $l=\left(g / 4 \pi^{2}\right) t^{2}$, where $t$ is the period of the pendulum.

Thus, event duration specifies spatial scale within the boundary conditions of each single event type. However, because the relations differ, mere event duration does not specify spatial scale if more than one event type is considered. McConnell et al. (1998) noted that the only difference among these relations is the value of the constant by which the square of duration is multiplied to obtain the spatial scale. This means that even if one considers several event types, the spatial scales of events are specified by event duration in combination with an event-specific constant.

McConnell et al. (1998) performed two experiments that investigated observers' abilities to use this information. Participants were asked to judge the distance and size of balls. In Experiment 1, which simulated balls in the different event types, participants seemed to be able to judge ball size, but the judgment variability (both within and among observers) was large. It was concluded that the participants were sensitive to the event durations and to the relations between the event-specific constants, but not to the precise values of the constants. In Experiment 2, the variability of distance judgments decreased after training with feedback on one event type, and this generalized to the other event types. The improvement of the distance judgments was interpreted as a calibration of the event-specific constants.

In the present observation, we argue that these conclusions are not justified and that other interpretations of the results are more appropriate. We claim that (1) observers' ability to accurately estimate distance and size does not necessarily imply the use of variables that specify the tobe-perceived property unless the use of nonspecifying variables can be ruled out, (2) analyzing data averaged over participants might lead to erroneous conclusions about the variables used by individualobservers, and (3) a decrease in within-observers variability in the judgments cannot be attributed to the calibration of event-specific constants. We will further explain these points in turn and then demonstrate their viability with an experiment in which participants are trained with feedback to judge the distance and size of falling balls.

\section{THE USE OF SPECIFYING VERSUS NONSPECIFYING VARIABLES}

In this article, we concentrate on the case of freely falling balls, because more experimental work has been done with this than with other event types. We limit ourselves to vertically falling balls (see Royden, 1994, and Tresilian, 1994, for a discussion about distance and size information for freely falling balls with a horizontal velocity component). In this section, we define the terms specifying and nonspecifying variables as they are used 
in this article, briefly review the evidence concerning observers' abilities to use specifying information, and conclude that in order to support the use of specifying information, one should rule out the possible use of nonspecifying variables.

A variable specifies a particular property if it is oneto-one related to the property. For instance, if a falling ball is initially stationary and not affected by air resistance, the height of the fall is specified by the duration of the fall. Given a particular height, the optical size of the falling event specifies the distance between the observer and the event, and the optical size of the ball relative to the optical size of the event specifies the size of the ball. Thus, the distance and size of a falling ball are specified by the duration of the fall in combination with image size. In addition, the distance of freely falling balls is specified by optical acceleration (Saxberg, 1987; Watson, Banks, von Hofsten, \& Royden, 1992), and the size of falling balls is specified by the time between the onset of motion and the moment at which the ball has fallen a distance equal to its optical size. In the present article we do not aim to distinguish among these specifying variables. Rather, we aim to reveal whether individuals use specifying or nonspecifying variables.

A variable does not specify a particular property if a single value of the variable can go together with several states of the property. For instance, the duration of a fall in itself does not specify the distance between the observer and the falling ball, because balls falling at different distances can have the same fall duration. It is important to realize, however, that a variable might correlate highly with a property, even if it does not specify the property. This can happen naturally or because of contrived experimental circumstances. For instance, if balls at a greater distance tend to fall from a greater height, as was the case with the stimuli in McConnell et al.'s (1998) experiments, the duration and height of the falls will be correlated. This means that, in such a situation, observers who base their distance judgments only on duration might show reasonably accurate performance, even though they use a nonspecifying variable.

Are observers able to use information that specifies the distance and size of vertically falling balls? Three studies provide tentative reasons to answer this question in the affirmative. First, Johansson and Jansson (1967) asked participants to adjust the speed of a film showing a continuous loop of an athlete engaged in diving, pole vaulting, or high jumping. The participants set the correct speed of the film with high accuracy. Second, $\mathrm{Mu}-$ chisky and Bingham (1992) reported that participants were able to perceive the simulated size of projected falling balls. Third, participants in an experiment by Stappers and Waller (1993) accurately adjusted the simulated vertical acceleration of the water of a computeranimated fountain so as to make the display look natural. The authors of these studies concluded that the observed performance can be explained by participants' use of specifying information.
However, this conclusion seems to conflict with a study by Hecht, Kaiser, and Banks (1996), who performed five experiments in which observers were asked to judge the distance and size of falling balls. It was found that performance was not diminished by variations in the simulated ball sizes and in the trajectory lengths on the screen. Hence, the judgments were not based on such variables as fall duration and optical size, because these variables vary over different trajectory lengths and simulated sizes. Hecht et al. also compared constant acceleration and constant velocity conditions; on these trials, balls either accelerated normally or fell at a constant speed. Performance did not differ significantly in these conditions, which led Hecht et al. to conclude that the participants used average optical velocity, rather than optical acceleration, because average velocity was available in both conditions.

Hecht et al.'s (1996) study demonstrates that just because performance is good, one cannot conclude that observers use specifying information; one must make sure that the observers do not use nonspecifying variables. In part, the experiments of McConnell et al. (1998) could not rule out the use of nonspecifying variables, because specifying and nonspecifying variables were confoundedthat is, the design of the experiment did not allow one to distinguish reliance on specifying information from reliance on crucial nonspecifying variables. As was argued by Hecht et al., different trajectory lengths on the screen should be used in the case of falling balls in order to unconfound specifying information and, for instance, the maximal image velocity. Similarly, different slope lengths on the screen would unconfound relevant variables in the case of rolling balls, and different angular excursions of the pendulum would do the same in the case of swinging balls. Because of the limited variation in displays, McConnell et al. could not distinguish reliance on specifying information from reliance on a number of nonspecifying variables that have been shown to be relevant, at least in the case of falling balls (Hecht et al., 1996).

Furthermore, the analyses of McConnell et al. (1998) did not consider possible nonspecifying variables that were unconfounded. For instance, the use of different inclines in their Experiment 1 unconfounded specifying information and the final image speed of rolling balls; the correlation between specifying information (or simulated size) and the (negative of) final image speed of rolling balls was .73 , which implies that the use of these variables could have been distinguished. ${ }^{3}$ Nevertheless, the use of final image speed could have led to correlations between judgments and size that were as high as .73. Thus, accurate performance by itself would have ruled out the use of final image speed only if the correlations between the judgments and size had been higher than .73. The participants in the experiments of McConnell et al. seemed to perform less accurately, however. Over all events, McConnell et al. reported correlations of .42 and .61 between size and the judgments of two groups of observers (for who different response techniques were 
used). Such levels of performance did not rule out the possible use of nonspecifying variables.

In summary, relevant nonspecifying variables should be considered before one can conclude that observers use specifying information. McConnell et al. (1998) considered only the use of information that specified the scales of all event types and the use of mere duration. Thus, one can conclude with certainty only that the use of specifying information explains more judgment variability than does the use of mere duration. Below, we present results that demonstrate that other nonspecifying variables, in turn, can explain more variability than does specifying information in the case of falling balls.

In addition, care must be taken in defining candidate optical variables. The duration of a falling event, for instance, can be defined as the time between the onset of motion and the moment at which a ball has fallen a distance equal to its diameter, as the time between the onset of motion and the moment a ball hits the ground, and so forth. The choice of a particular definition is important, because duration specifies the size of the ball if the first definition is used, but it specifies the height of the fall if the second definition is used. Similarly, the duration of a pendulum event can be defined as the period of a pendulum or as half a period. McConnell et al. (1998) used the first definition. They reported significant differences in how duration was used in the different event types (see their Figure 4). We suspect that some of these differences would disappear if the second definition were to be used; in that case, the size judgment in the pendulum event might not be associated with longer durations than in the other events.

\section{INDIVIDUAL DIFFERENCES IN VARIABLE USE}

In the previous section, we argued that observers' ability to use information that specifies distance and size is an empirical issue and, hence, that the possible use of nonspecifying variables should be considered. Here, we provide similar arguments with respect to individual differences. The idea that all individuals use the same variables follows both from assumptions of specificity in the theory of direct perception (e.g., Burton \& Turvey, 1990) and from assumptions by heuristics theorists that observers are limited to the use of a few dimensions of information and obey certain salience rules (see, e.g., Gilden \& Proffitt, 1989). Hence, experimenters studying the perception of distance and size of falling balls and of the length of pendulums (Pittenger, 1985, 1990) usually presented their results averaged over observers.

However, it has recently been demonstrated that observers differ in the kinematic variables they use for the visual perception of relative pulling force (Michaels \& de Vries, 1998) and relative mass of colliding balls (Jacobs, Michaels, \& Runeson, 2000). Different observers might also use different variables for the perception of distance and size. Thus, averaging over observers may lead to erroneous conclusions about the used variables. In addition, Michaels and de Vries and Jacobs et al. showed that observers changed in the variables they used after training with feedback. In the next section, we propose that the decrease in within-observers variability reported by McConnell et al. (1998) can be attributed to changes in the variables used.

\section{CHANGES IN VARIABLE USE}

In their Experiment 2, McConnell et al. (1998) used feedback to train observers to make judgments of the distance of balls rolling down inclines. After training, observers were asked to judge the distance of falling, rolling, and swinging balls. The average correlation between the judged and the simulated distances of rolling balls improved from .50 before training to .89 after training, and the improvement generalized to the other event types. McConnell et al. suggested that the decrease in variability might have been due to the calibration of event-specific constants. They claimed that observers are always sensitive to the appropriate relations between the physical constants, but not to their precise values. Observers become attuned to the precise values of the physical constants-without changing the relations between the used constants - only after practice with feedback.

This interpretation predicts that the relations between the constants that observers used after training are equal to the relations between the physical constants and that the values correspond to the physical values. The experiment confirmed these predictions (see Figure 11 and Tables 5 and 6 of McConnellet al., 1998). Unfortunately, McConnell et al. did not test the further predictions of their interpretation. They could have tested whether, before training and in Experiment 1, the relations between the constants used were equal to the relations between the physical constants and whether the precise values were different from the physical values. Thus, McConnell et al. demonstrated that the hypothesized constants were well calibrated after training, but not that they were calibrated less well before training.

Of course, this does not suggest that the calibration did not occur. However, even if the calibration occurred, it cannot explain the decrease in within-observers variability. This variability was measured with correlations, and correlations are insensitive to multiplication by a constant. Hence, the decrease in within-observers variability is still in need of explanation. We propose that it might be understood, at least in part, as a change in which variables observers use. Michaels and de Vries (1998) and Jacobs et al. (2000) showed that observers change in the kinematic variables they use for the perception of kinetic properties. Before training, observers tend to use variables that correlate only moderately with 
to-be-perceived properties. After training with feedback, observers come to rely on variables that correlate more highly with the to-be-perceived properties or even on specifying information. Below, we demonstrate that this process of convergence on the more useful variables also occurs in the perception of the distance and size of falling balls.

\section{EXPERIMENT}

We have suggested that observers might use nonspecifying variables for the perception of distance and size, that observers might differ from each other in the variables they use, and that they might converge on the more useful variables. The purpose of the present experiment was to test these predictions. Observers were trained with feedback to judge the distance and size of videotaped falling balls. ${ }^{4}$ We considered the possible use of distance- and size-specifying information, fall duration, optical size, and peak velocity. Fall duration was defined as the time between the onset of motion and the moment at which a ball disappears from the screen, and peak velocity as the maximal velocity of the ball on the screen.

Different trajectory lengths and simulated ball sizes were used to unconfound nonspecifying variables from variables that specify the simulated distance and size. The correlations among the candidate optical variables are presented in Table 1. All the correlations are considerably less than 1.0, which indicates that reliance on the different variables can be distinguished. On the basis of the results of Michaels and de Vries (1998) and Jacobs et al. (2000), we expected that observers would start with different variables, would change which variables they relied on after practice and, perhaps, eventually would converge on the use of specifying information.

\section{Method}

Participants. Five male and 5 female students of the Faculty of Human Movement Science were paid for their participation.

Apparatus. White balls of different sizes were made to fall at different accelerations against a dark background. To create the different accelerations, the balls were suspended by a string that went by pulleys behind a background curtain. Various masses were attached to the end behind the curtain. The string thereby exerted an upward force on the balls proportional to the counterweight. Thus, the balls accelerated at $9.8 *\left(\right.$ mass $_{\mathrm{w}}-$ mass $\left._{\mathrm{b}}\right) /\left(\right.$ mass $_{\mathrm{w}}+$ mass $\left._{\mathrm{b}}\right)$ $\mathrm{m} / \mathrm{sec}^{2}$. The balls were filmed with a VHS video camera and presented on a $54-\mathrm{cm}$-diameter screen at $1 \mathrm{~m}$ from the participants. During the experiment, the intensity of the screen was set so that only the balls were visible. The videotapes were digitized in order to obtain the precise values of the variables considered. Ten markers were placed between 2 and $20 \mathrm{~m}$ down the hall where the experiment was run, and nine disks, which varied in diameter from 4 to $34 \mathrm{~cm}$, were placed above the screen; each was labeled clearly with its distance or size to serve as reference distances and sizes for participants' judgments.

Design. Forty-five trajectories were used. The simulated distances ranged from 3 to $33 \mathrm{~m}$, and the simulated sizes ranged from 4 to $78 \mathrm{~cm}$. The balls always started stationary near the top of the screen and disappeared after falling screen distances of 8,16 , or $24 \mathrm{~cm}$. The observers were shown two series of three blocks. Both started with Block 1 without feedback, followed by Blocks 2 and 3 with feedback. The same stimuli were used in Blocks 1 and 3. Observers 1-5 started with three blocks of distance judgments, followed by three blocks of size judgments. Observers 6-10 started with the size judgments.

Procedure. On any trial, a ball trajectory was shown on the video monitor. During a 10 -sec interval that separated the trials, the observers verbally reported the size or the distance of the balls. They were asked to give a numerical estimate of the simulated size or distance of the ball based on the values given on the markers in the hall or in the disks on the wall. The instructions clearly indicated that the simulated distance and size might be outside the range of the samples. In feedback trials, the experimenter verbally informed the participant about the correct distance or size immediately after a judgment. There was a short break between the two sets of three blocks, and the total experiment lasted about $45 \mathrm{~min}$.

\section{Results}

We first present analyses that test whether the participants were able to perform the task and whether they improved after practice with feedback. The left panel of Figure 1 shows the judged distances as a function of the simulated distances, averaged over all the participants. In the first block, the participants overestimated short distances and, more dramatically, underestimated long distances. In the second and third blocks, in which feedback was given, both the overestimation and the underestimation diminished; distance perception seemed to improve after practice with feedback. Similarly, the right panel of Figure 1 shows that size perception improved with feedback.

To determine whether variation in the judgments corresponded with variation in the simulated distance and size, we calculated the Pearson product moment correlations between the judged and the simulated metrics for each block of trials of each observer. These correlations are presented as parts of Figures 2 and 3 (filled dots and open triangles). The correlations between the judged and the simulated distances differed significantly $(p<.05)$ from zero in 22 (of 30) blocks, and the correlations between the judged and the simulated sizes differed signif-

Table 1

Correlations Among Candidate Optical Variables

\begin{tabular}{llllrl}
\hline \multicolumn{1}{c}{ Variable } & 1 & 2 & 3 & 4 & \multicolumn{1}{c}{5} \\
\hline 1. Distance-specifying information & - & .71 & .81 & -.16 & -.75 \\
2. Size-specifying information & & - & .50 & .49 & -.66 \\
3. Duration of fall & & & - & -.23 & -.43 \\
4. Optical size & & & & - & -.02 \\
5. Peak velocity & & & & - \\
\hline
\end{tabular}



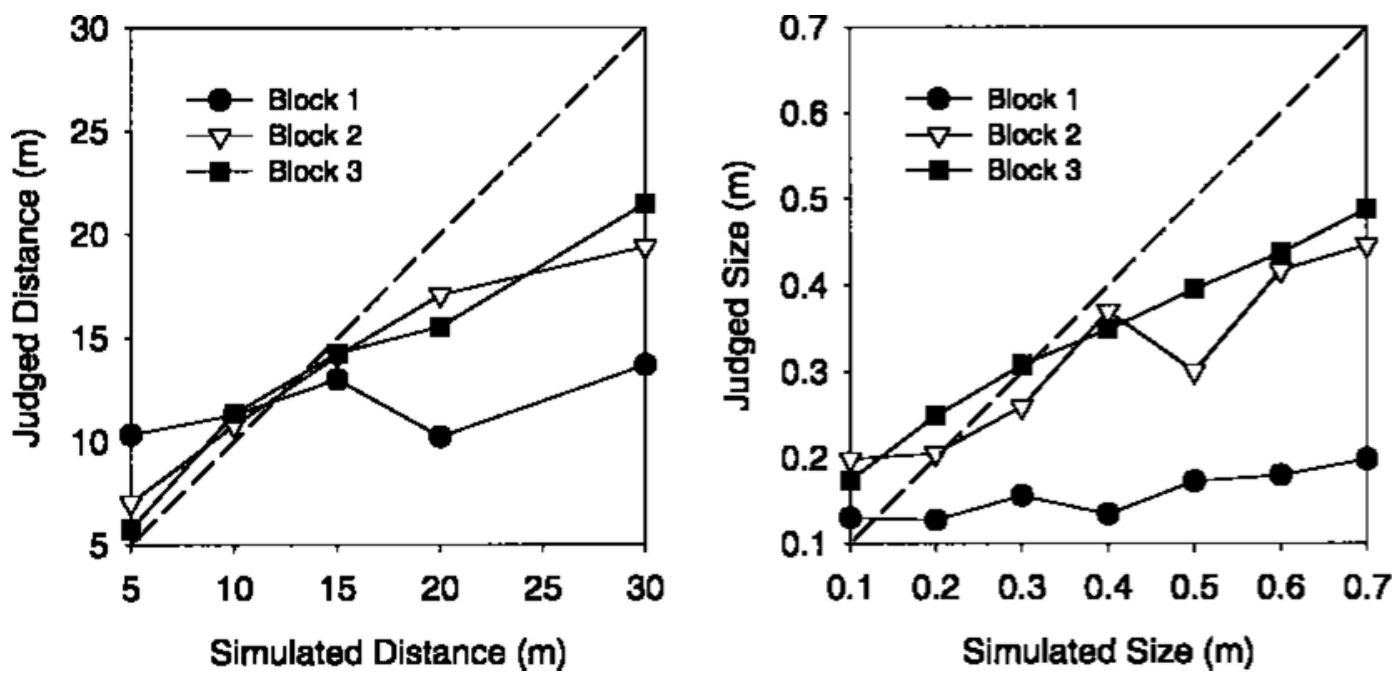

Figure 1. Left panel: Averaged judged distances as a function of simulated distances for the three blocks of trials. Right panel: Averaged judged ball sizes as a function of simulated sizes, again for each block of trials.

icantly ( $p<.05$ ) from zero in 23 (of 30) blocks. Thus, the observers mostly appeared to be able to perceive distance and size.

We performed two analyses of variance on these correlations, with block of trials $(1,2$, and 3$)$ as a withinsubjects factor, one for the distance judgments and one for the size judgments. 5 The effect of block was significant in both analyses $[F(2,18)=24.0, p<.001$, and $F(2,18)=8.7, p<.01]$. In agreement with the findings of McConnell et al. (1998), judgments of distance and size improved after practice with feedback. In the following, we address which variables were used and whether the participants differed and changed in the variables they used.

To determine the variables on which the participants relied, we calculated the correlations between the judgments and the variables listed in Table 1. Figure 2 presents the squares of these correlations for each block of trials in which the participants were asked to judge distance, and Figure 3 for the blocks in which size judgments were made. The letters above the columns of points indicate whether the differences among the correlations were significant according to a $t$ test for independent correlations (Bruning \& Kintz, 1987).

In the first block of distance judgments, a number of the participants $(1,2,4,5$, and 9$)$ seemed to use optical size (shown by open diamonds). Optical size had essentially no correlation with distance $(r=-.16)$, because of the variation in simulated ball size. Hence, judgments based on optical size correlated poorly with the distances and resulted, in the later blocks, in feedback indicating poor performance. Perhaps because of the feedback, most of these participants changed in the variables on which they relied. Participant 1 , for instance, appeared to come to rely on fall duration (filled squares). Duration correlated reasonably with distance $(r=.81)$, despite the differences in trajectory lengths, and its use apparently resulted in acceptable performance.

For many blocks of trials, firm statements about the variables used could not be made, because the differences did not reach significance. Nevertheless, in the majority of blocks, variables could be ruled out as the optical basis of perception. In the first block, the use of distance-specifying information could be ruled out for Participants 1, 2, 4, 5, 8, and 9 because other variables explained significantly more variance, and for Participant 3 because it did not appear to correlate with the judgments. Furthermore, the differences that were significant and the nominal correlations suggested some patterns. Participants 8 and 10, for instance, seemed to rely on peak velocity in the first block and on distancespecifying information in the later blocks. In sum, we found differences and changes in the variables that were used in the perception of distance. Optical size seemed to be used in the first blocks, but not in the later blocks. Finally, the use of distance-specifying information could, for the majority of the participants, be ruled out in the first block, but not in the second and third blocks.

In the first block of size judgments, Participants 7, 9, and 10 seemed to use optical size. Participant 9 persevered with the use of optical size in Blocks 2 and 3, but the judgments of Participant 7 and, less clearly, Participant 10 correlated higher with simulated size (open triangles) in the later blocks, indicating that these observers came to rely on size-specifying information. For the other participants, the candidate variables were difficult to distinguish in the first block. However, in the third block, the judgments of Participant 1 seemed to be based on duration, and the judgments of Participants 2, 3 , and 5 on size-specifying information. In sum, we also found individual differences and convergence on the more useful variables in the perception of size. In the third 

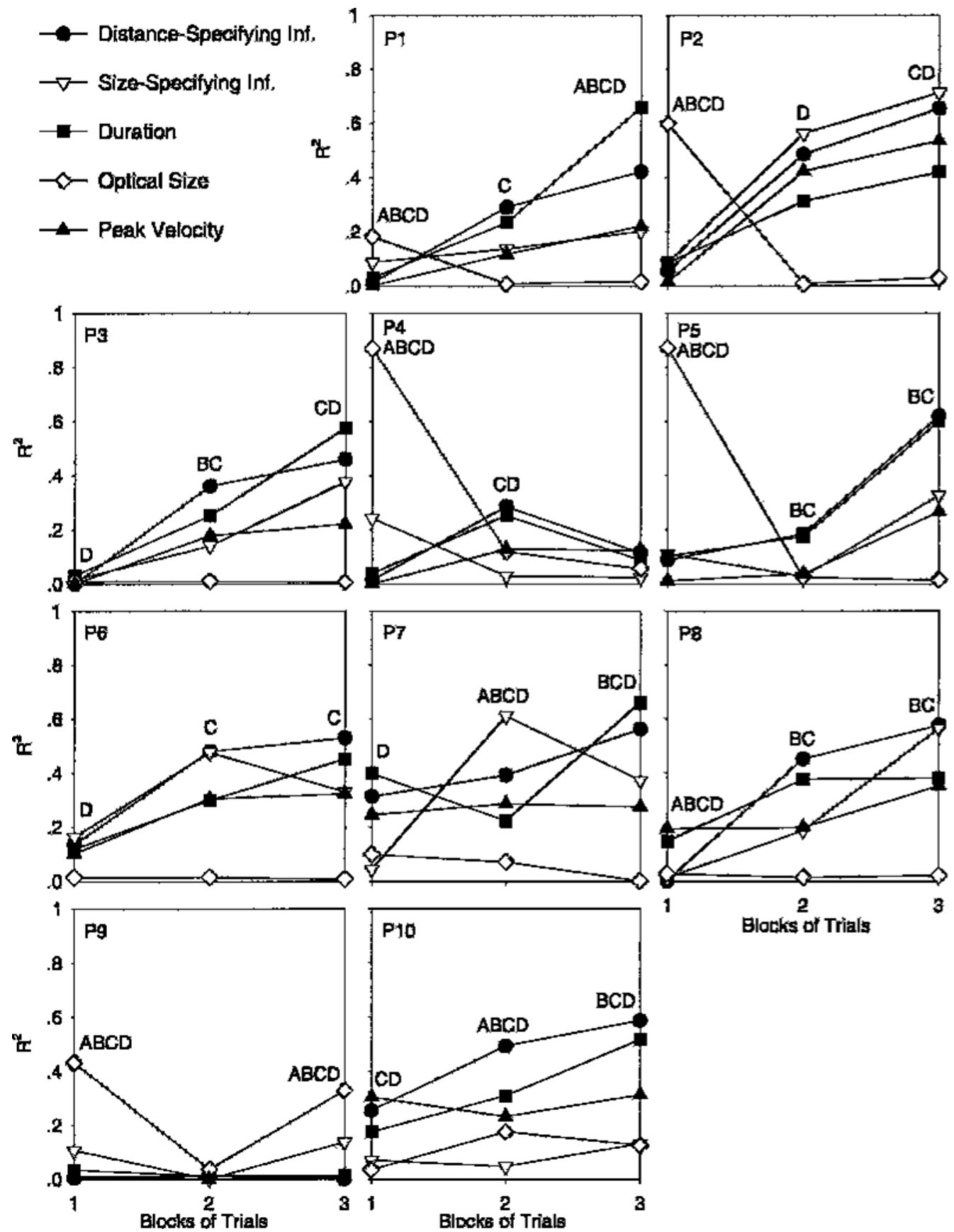

Blocks of Trials

Figure 2. Squares of correlations between the various optical variables and the judged distances for all the participants. In columns marked with an A, B, C, and D, the highest correlation was significantly different $(p<$ .05) from, respectively, the second, third, fourth, and fifth highest correlation.

block, 4 participants seemed to rely on size-specifying information.

\section{Discussion}

The participants in the present experiment were trained with feedback to judge the distance and size of freely falling balls. We determined, observer by observer and block by block, which optical variables explained most of the systematic variance in the judgments. It was found that performance improved after practice with feedback and that observers differed in the variables they used. Before practice, most observers used variables that are not specific to distance and size. With practice, observers changed in the variables they used for the percep- 

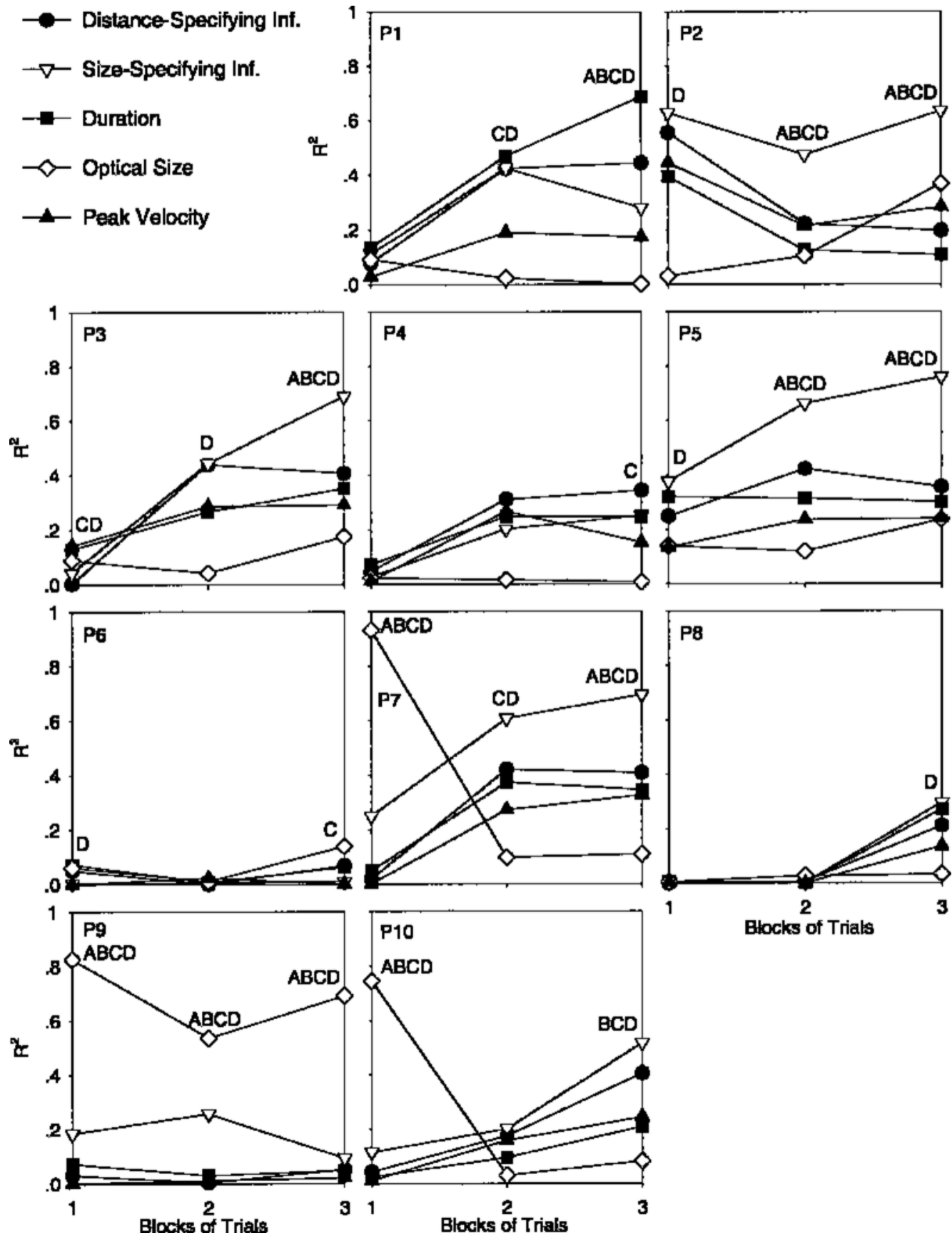

Blocks of Trials

Figure 3. Squares of correlations between the various optical variables and the judged sizes for all the participants. In columns marked with an $\mathrm{A}, \mathrm{B}, \mathrm{C}$, and $\mathrm{D}$, the highest correlation was significantly different $(p<.05)$ from, respectively, the second, third, fourth, and fifth highest correlation.

tion of size; several observers appeared to discover sizespecifying information. Observers also changed in the variables they used for the distance judgments; in general, they came to rely on the more useful ones. However, few observers appeared to discover distance-specifying information.
The finding that many observers seemed to rely on nonspecifying variables, most markedly before practice, shows that analyses that aim to reveal the optical bases of perception should seriously consider the possible use of nonspecifying variables. The use of nonspecifying variables is in agreement with the conclusions of Hecht 
et al. (1996). These authors reported that judgments of the distance and size of freely falling balls are not based on optical acceleration-information that specifies the distance of falling balls. Nevertheless, after practice, the distance judgments of a few participants in the present experiment seemed to be based on specifying information, and reliance on specifying information could not be ruled out for many others. Our methodology did not allow us to determine which type of specifying information these observers used. They could have used, for instance, optical acceleration or the duration of the falls in combination with image size.

One could argue that optical acceleration would be more useful for the perception of distance. First, acceleration specifies the distance of falling balls in a wider range of situations; unlike duration in combination with image size, it does not require a ball to start stationary. Second, acceleration is available at any moment of the fall, whereas duration requires the perceiver to observe the event from the beginning to the end. Third, acceleration specifies the to-be-perceived distance and can thus be used for the direct perception of distance. Duration, in contrast, specifies only the height of the fall and requires further processing to infer the distance of the falling ball. On the other hand, several studies question observers' abilities to detect optical acceleration (cf. Calderone \& Kaiser, 1989; Gottsdanker, Frick, \& Lockard, 1961; Schmerler, 1976; although see also Babler \& Dannemiller, 1993; Rosenbaum, 1975).

The finding that individuals differ in the variables they use reaffirms that analyses that aim to reveal the optical basis of perception should be performed observer by observer. In the case of size judgments, for instance, some participants seemed to rely on optical size (e.g., Participant 9), some seemed to rely on specifying information (e.g., Participant 2), and some seemed to change from the use of optical size to reliance on specifying information (e.g., Participant 7). Similarly, Michaels and de Vries (1998) and Jacobs et al. (2000) reported that different individuals use different kinematic variables in the perception of kinetic properties. Given these differences, analyses on averaged data may lead to erroneous conclusions.

These results have important implications for the recent article of McConnell et al. (1998), which reported that the participants were able to judge the distance and size of balls in different event types. It was claimed that the judgments were based on event duration in combination with event-specific constants (which specified the spatial scales of the different event types), rather than on mere duration. McConnell et al. analyzed their data mainly averaged over observers, and they considered only variables that specified spatial scale-either over all event types or under the more restricted boundary conditions of a single event type. Such analyses are appropriate if all observers use the same variable and if observers do not use nonspecifying variables. In our study, this did not appear to be the case. Hence, we argue that the participants in the experiments of McConnell et al. also might have differed in the variables they used and that they might have used nonspecifying variables.

Finally, in agreement with the findings of McConnell et al. (1998), we found that performance improved after practice with feedback. More specifically, the correlations between the simulated properties and the judgments increased. These correlations might have increased because observers came to use variables that correlated more highly with the to-be-perceived properties. McConnell et al. claimed that the improvement was due to calibration of event-specific constants. However, we have argued in the introduction that the decrease in within-observers variability cannot be explained by calibration of the constants. Note that we do not claim that calibration did not occur. Calibration might have contributed to other improvements of performance, such as the decrease in between-observers variability or the decrease in proportional error (see Figure 4 of McConnell et al., 1998).

To conclude, we want to emphasize that we agree with McConnell et al. (1998) that experiments concerning a single event type (including the present one) confound variables that specify the spatial scales of different event types with variables that specify only the spatial scale of a single event type. In their experiments, McConnell et al. considered different event types and, hence, unconfounded these variables. This led to many interesting questions. For instance, do observers rely on different variables in different event types? Under which conditions does fostered reliance on a certain variable generalize to different event types? Or, can observers come to use information that specifies distance and size in all event types? In our view, such questions remain to be answered by future research.

\section{REFERENCES}

Babler, T. G., \& DAnnemiller, J. L. (1993). Role of image acceleration in judging landing location of free-falling projectiles. Journal of Experimental Psychology: Human Perception \& Performance, 19, 15-31.

Bruning, J. L., \& Kintz, B. L. (1987). Computational handbook of statistics. Glencoe, IL: Scott Foresman.

Burton, G., \& Turvey, M. T. (1990). Perceiving the lengths of rods that are held but not wielded. Ecological Psychology, 2, 295-324.

Calderone, J. B., \& Kaiser, M. K. (1989). Visual acceleration detection: Effect of sign and motion orientation. Perception \& Psychophysics, 45, 391-394.

Edgar, G. K., \& Bex, P. J. (1995). Vision and displays. In K. Carr \& R. England (Eds.), Simulated and virtual realities: Elements of perception (pp. 85-101). London: Taylor \& Francis.

Gilden, D. L., \& Proffitt, D. R. (1989). Understanding collision dynamics. Journal of Experimental Psychology: Human Perception \& Performance, 15, 372-383.

GotTsdanker, R. Frick, J. W., \& LockArd, R. B. (1961). Identifying the acceleration of visual targets. British Journal of Psychology, 52, 31-42.

Hecht, H., Kaiser, M. K., \& Banks, M. S. (1996). Gravitational acceleration as a cue for absolute size and distance? Perception \& Psychophysics, 58, 1066-1075.

Jacobs, D. M., Michaels, C. F., \& Runeson, S. (2000). Learning to perceive the relative mass of colliding balls: The effects of ratio-scaling and feedback. Perception \& Psychophysics, 62, 1332-1340. 
Johansson, G., \& JANSSON, G. (1967). The perception of free fall. Unpublished seminar paper, University of Uppsala, Department of Psychology.

McConnell, D. S., Muchisky, M. M., \& Bingham, G. P. (1998). The use of time and trajectory forms as visual information about spatial scale in events. Perception \& Psychophysics, 60, 1175-1187.

Michaels, C. F., \& DE VRIEs, M. M. (1998). Higher-order and lowerorder variables in the visual perception of relative pulling force. Journal of Experimental Psychology: Human Perception \& Performance, 24, 526-546.

Muchisky, M. M., \& Bingham, G. P. (1992). Perceiving size in events via kinematic forms. In J. Kruschke (Ed.), Proceedings of the 14th Annual Conference of the Cognitive Science Society (pp. 1002-1007). Hillsdale, NJ: Erlbaum.

Pittenger, J. B. (1985). Estimation of pendulum length from information in motion. Perception, 14, 247-256.

Pittenger, J. B. (1990). Detection of violations of the law of pendulum motion: Observers' sensitivity to the relation between period and length. Ecological Psychology, 2, 55-81.

Rosenbaum, D. A. (1975). Perception and extrapolation of velocity and acceleration. Journal of Experimental Psychology: Human Perception \& Performance, 1, 395-403.

Royden, C. S. (1994). In defense of "Gravity as a monocular cue for perception of absolute distance and/or absolute size." Perception, 23, 733-735.

SAXBerg, B. V. H. (1987). Projected free fall trajectories: 1. Theory and simulation. Biological Cybernetics, 56, 159-175.

Schmerler, J. (1976). The visual perception of accelerated motion. Perception, 5, 167-185.

Stappers, P. J., \& Waller, P. E. (1993). Using the free fall of objects under gravity for visual depth estimation. Bulletin of the Psychonomic Society, 31, 125-127.

Tresilian, J. R. (1994). A note on "Gravity as a monocular cue for perception of absolute distance and/or size." Perception, 23, 727-731.

Watson, J. S., Banks, M. S., von Hofsten, C., \& Royden, C. S. (1992). Gravity as a monocular cue for perception of absolute distance and/or absolute size. Perception, 21, 69-76.

\section{NOTES}

1. We are much less enthusiastic than McConnell et al. (1998) about the usefulness of the duration of rolling events in setting spatial scale. Although McConnell et al. acknowledged the effect of mass distribution on acceleration down a plane, it was not mentioned in association with the equation that described the rolling event. Mass distribution has a strong effect on acceleration. To take extremes, a sphere with all the mass concentrated at the center would accelerate down a $30^{\circ}$ plane at $4.9\left(\mathrm{~m} / \mathrm{sec}^{2}\right)$, a sphere with homogenous mass distribution at 3.5 $\left(\mathrm{m} / \mathrm{sec}^{2}\right)$, a hollow thin-walled sphere at $2.9\left(\mathrm{~m} / \mathrm{sec}^{2}\right)$, and a thin-walled hollow cylinder at $2.5\left(\mathrm{~m} / \mathrm{sec}^{2}\right)$. In short, spatial scale and rotational inertia both affect image acceleration of balls rolling down a plane. Add to that the fact that sliding becomes more likely as the pitch increases, and one concludes that, in normal circumstances, perceivers should not be able to accurately set spatial scale on the basis of rolling events.

2 . Note that this equation differs by a factor of two from the equation presented by McConnell et al. (1998).

3 . To compute this correlation, we first calculated the simulated final speeds $(v)$, using the formula $v=\sqrt{ }\left(1.43^{*} h^{*} g\right)$, in which $h$ is the simulated vertical distance covered by a (solid, homogenous) ball and $g$ the gravitational constant. Knowing the simulated final speeds on each trial, one can easily calculate the final speeds on the screen and then the correlation between these speeds and the simulated sizes.

4. We preferred to use video, rather than computer simulations, to reduce aliasing (see, e.g., Edgar \& Bex, 1995). The presentation of sharp images in successive frames does not seem to optimally stimulate detection mechanisms. If this is so, this could lead erroneously to the conclusion that observers are not able to detect information. Previous failures to find support for the use of optical acceleration (e.g., Hecht et al., 1996) might have been due to such imperfections in the simulations.

5 . All the tests on correlations were done on the $Z$ transformations of the correlations, $Z_{r}=1 / 2[\ln (1+r)-\ln (1-r)]$.

(Manuscript received September 18, 1999; revision accepted for publication June 29, 2000.) 